\title{
Evaluation of Quality Characteristics of Panzhihua Moringa Oleifera
}

\author{
Lijun Zhang1, a , Xingguo Zhang', b and Jun Zhang1, c, * \\ ${ }^{1}$ School of Life Science, Beijing Institute of Technology, Beijing 100081, China. \\ ${ }^{2}$ School of Life Science and Engineering, Southwest Jiao tong University, Chengdu 611756, China. \\ azljrenzhe@163.com, ${ }^{b}$ zxg332@126.com, ${ }^{*}, c$ zhangjun@bit.edu.cn
}

Keywords: Maringa Oleifera; Content Determination; Quality Characteristics.

\begin{abstract}
In order to provide a scientific basis for the development and utilization of Maringa, dry powder of Maringa leaves and seeds are used as raw materials, referring to the relevant methods in Chinese Pharmacopoeia (2015 edition) and literatures, the contents of moisture, ash and extracts of Maringa oleifera were determined. The results showed that the moisture content of Maringa leaves and of Maringa seeds were $4.19 \%$ and $3.47 \%$, the water extracts were $36.28 \%$ and $19.19 \%$, the alcohol extracts were $24.85 \%$ and $13.70 \%$, the total ash were $11.04 \%$ and $3.57 \%$, the acid insoluble ash were $2.18 \%$ and $0.30 \%$. The content of total polysaccharides and total flavonoids of Maringa leaves were $11.14 \%$ and 3.7\%, respectively. The content of oil of the Maringa seeds was $22.70 \%$. The present study could provide experimental basis and help for evaluating the quality of Maringa, and developing related functional products.
\end{abstract}

\section{Introduction}

Moringa oleifera is a tropical plant that belongs to the monogenetic family Moringaceae $[1,2]$. Recent studies find that Moringa leaves and seeds contain a lot of nutrients and active ingredients, showing a series of important medicinal value and health care functions such as lowering blood pressure, preventing cardiovascular diseases, improving immunity of the body and inhibiting tumors [3]. Among them, polysaccharides and flavonoids are the main active ingredients of Moringa leaves, oil is the major component of Maringa seeds [4, 5]. At present, the literature and research about Moringa are mostly about its nutritional value, but very few experimental studies on the determination of active ingredients and the comprehensive quality evaluation were conducted. Using the Panzhihua Moringa, this work aims to provide experimental basis for the quality characteristics study of Moringa. Specificly, referring to the Chinese Pharmacopoeia(ChP, 2015 edition) [6] and related literatures [7-10], the contents of moisture, ash, water extracts, alcohol extracts, oil, total polysaccharides and total flavonoids were detected.

\section{Experimental}

\subsection{Materials.}

Chemicals and reagents. The same batch of fresh Moringa leaves and Moringa seeds were collected from Panzhihua Moringa cultivation base; The standard glucose and rutin were purchased from NIFDC (Nation Institutes for Food and Drug Conrol, China), they were used to quantify total polysaccharides and total flavonoids of Moringa leaves, respectively; Ethanol, chloroform, n-Butanol, sulfuric acid, petroleum ether, anthrone (9,10-dihydro-9-oxo-anthracen) and acetone were of HPLC grade and purchased from Beijing Chemical Works.

Sample pretreatment.Place the Moringa leaves and seeds in an oven at $60^{\circ} \mathrm{C}$ to dry, then crush and seal.

\subsection{Instrumentation.}

Electric thermostatic drier (Suzhou Binde Oven Manufacturng Co., Ltd, Suzhou, China). Electronic balance (e $=10 \mathrm{~d}$, Beijing Sartorius Instrument System Co., Ltd, Beijing, China). Resistance furnace (Carbolite Gero, Beijing, China). Electric stove (Beijing Ever Bright Medical 
Treatment Instrument Co., Ltd, Beijing, China). DK-98-1 electric heating constant temperature water-bath (Tianjin Tesis Instrument Co., Ltd, Tianjin, China). SK7200LH Ultrasonic cleaner (Su Zhou FEAT Untrasonic Co, Ltd, Suzhou, China). TU-1901 UV/Visible spectrophotometer (Shanghai Aucy Technology Instrument Co., Ltd, Shanghai, China), et al.

\subsection{Routine Quality Assessment.}

According to the relevant standards of Chinese Pharmacopoeia appendix (Chp, 2015 edition) [6] , a certain amount of dry powder of Moringa leaves and seeds was used, the content of moisture, ash (total ash and acid insoluble ash), water extracts and alcohol extracts were measured (six replication each).

\subsection{Determination of Total Polysaccharide of Meringa Leaves.}

Preparation of polysaccharide sample.1.0g powder of the Moringa leaves was first weighted (six replication), then water extraction and alcohol precipitation method [8] was utilized to extract the crude polysaccharide. Finally, the polysaccharide extraction solution was diluted to $250 \mathrm{~mL}$ as a sample solution with distilled water.

Preparation of calibration standards. The stock solution $(1.0 \mathrm{mg} / \mathrm{mL})$ of glucose was prepared by dissolving $0.1 \mathrm{~g}$ glucose in $100 \mathrm{~mL}$ distilled water. Calibration work solutions were prepared by diluting the stock solution into a final concentration series of $1.0 \mathrm{mg} / \mathrm{mL}, 0.9 \mathrm{mg} / \mathrm{mL}, 0.8 \mathrm{mg} / \mathrm{mL}$, $0.6 \mathrm{mg} / \mathrm{mL}, 0.4 \mathrm{mg} / \mathrm{mL}, 0.2 \mathrm{mg} / \mathrm{mL}$ and $0.1 \mathrm{mg} / \mathrm{mL}$ with diluted water, all solutions have 3 replicates.

UV/Visible spectrophotometric analysis. The UV/Visible spectrophotometric analysis of polysaccharide was conducted according to the method of Chen et al [8]. Specifically, $1 \mathrm{~mL}$ of sample or calibration solution was added to a $10 \mathrm{~mL}$ clean tube, followed by $4 \mathrm{~mL}$ of anthrone-sulfuric acid solution. Then the tube was placed in boiling environment for $10 \mathrm{~min}$ of reaction and stopped under ice-water conditions, the absorbance was measured by TU-1901 UV/Visible spectrophotometer at $620 \mathrm{~nm}$. Finally, the calibration curve was constructed via linear regression, and the polysaccharide content in the samples was calculated based on the glucose standard calibration curve.

\subsection{Determination of Total Flavonoids of Maringa Leaves.}

Preparation of flavonoids sample.5.0g powder of the Moringa leaves was first weighted (six replication), then ethanol extraction method [10-11] was used to extract the total flavonoids. Finally, the total flavonoids extraction solution was diluted to $100 \mathrm{~mL}$ as a sample solution with $70 \%(\mathrm{v} / \mathrm{v})$ ethanol.

Preparation of calibration standards. The stock solution $(200 \mu \mathrm{g} / \mathrm{mL})$ of rutin was prepared by dissolving $0.02 \mathrm{~g}$ rutin in $100 \mathrm{~mL} 60 \%(\mathrm{v} / \mathrm{v})$ ethanol. Calibration work solutions were prepared by diluting the stock solution into a final concentration series of $8.0 \mu \mathrm{g} / \mathrm{mL}, 7.0 \mu \mathrm{g} / \mathrm{mL}, 6.0 \mu \mathrm{g} / \mathrm{mL}$, $5.0 \mu \mathrm{g} / \mathrm{mL}, 4.0 \mu \mathrm{g} / \mathrm{mL}, 3.0 \mu \mathrm{g} / \mathrm{mL}, 2.0 \mu \mathrm{g} / \mathrm{mL}$ and $1.0 \mu \mathrm{g} / \mathrm{mL}$ with $30 \%(\mathrm{v} / \mathrm{v})$ ethanol, all solutions have 3 replicates.

UV/Visible spectrophotometric analysis. The UV/Visible spectrophotometric analysis of total flavonoids was conducted according to the method of Sun et al [10]. Specifically, $5 \mathrm{~mL}$ of sample or calibration solution was added to a $10 \mathrm{~mL}$ clean tube, then the $0.5 \mathrm{~mL}$ of $\mathrm{NaNO} 2,10 \%(\mathrm{v} / \mathrm{v}) \mathrm{Al}(\mathrm{NO} 3)$ and $4 \% \mathrm{NaOH}(\mathrm{v} / \mathrm{v})$ solution were added sequentially, after $20 \mathrm{~min}$ of reaction, the absorbance was measured by TU-1901 UV/Visible spectrophotometer at 510nm. Finally, the calibration curve was constructed via linear regression, and the total flavonoids content in the samples was calculated based on the rutin standard calibration curve.

\subsection{Determination of Oil of Maringa Seeds.}

Oil from Moringa seeds was extracted by ultrasonic method and measured by weight [12-13]. In particular, accurately weighed $1.0 \mathrm{~g}$ powder of the Moringa seeds in a conical flask (six replication), 20 times the amount of petroleum ether was added and the total weight was weighted, after 40min extraction in a $40 \mathrm{HZ}$ ultrasonic cleaner, the supernatant was collected and the above process was repeated twice. Finally, the extracts were combined, concentrated, oven dried $\left(60^{\circ} \mathrm{C}\right)$ and weighted sequentially, the content of oil in the seeds samples were calculated based on the dried extract weight. 


\subsection{Data Processing.}

The calibration curve was drawn with GraphPad Prism software (Version 6.0, Demo, GraphPad Software, USA), and the calculation of other content data was done with Microsoft Excel (Excel version in Microsoft Office 2016 for Windows).

\section{Results and Discussion}

\subsection{Routine Quality Assessment.}

The contents of moisture, ash, water extracts and alcohol extracts from Moringa leaves and seeds were shown in Table 1 - Table3.

Table 1. Moisture content of Maringa leaves and seeds $(n=6)$

\begin{tabular}{cccc}
\hline Material & Mean $(\%)$ & SD & RSD (\%) \\
\hline Maringa leaves & 4.19 & 0.08 & 1.86 \\
Maringa seeds & 3.47 & 0.06 & 1.80 \\
\hline
\end{tabular}

Table 1 shows that the moisture content of dried Moringa leaves and seeds was $4.19 \%$ and 3.47\%, respectively. The leaves was slightly higher than that of seeds.

Table 2. Ash content of Moringa leaves and seeds $(n=6)$

\begin{tabular}{cccccccc}
\hline \multirow{2}{*}{ Material } & \multicolumn{3}{c}{ Total ash } & & \multicolumn{3}{c}{ Acid insoluble ash } \\
\cline { 2 - 3 } \cline { 6 - 8 } & Mean (\%) & SD & RSD (\%) & & Mean (\%) & SD & RSD (\%) \\
\hline Maringa leaves & 11.04 & 0.16 & 1.45 & & 2.18 & 0.03 & 1.19 \\
Maringa seeds & 3.57 & 0.05 & 1.40 & & 0.30 & 0.01 & 3.33 \\
\hline
\end{tabular}

The results of Table 2 indicate that the total ash and acid insoluble ash of Moringa leaves are higher than those in seeds, especially the acid insoluble ash. Excluding the reason for the introduction of sediment and other impurities due to incomplete cleaning, the results show that the leaves of Moringa are more likely to enrich non-oxides such as inorganic salts and mineral elements.

Table 3. Extracts content of Moringa leaves and seeds ( $n=6)$

\begin{tabular}{cccccccc}
\hline \multirow{2}{*}{ Material } & \multicolumn{3}{c}{ Water extracts } & & \multicolumn{3}{c}{ Alcohol extracts } \\
\cline { 2 - 4 } \cline { 6 - 8 } & Mean $(\%)$ & SD & RSD (\%) & & Mean (\%) & SD & RSD (\%) \\
\hline Maringa leaves & 36.28 & 0.38 & 1.05 & & 24.85 & 0.15 & 0.60 \\
Maringa seeds & 19.19 & 0.30 & 1.56 & & 13.70 & 0.12 & 0.88 \\
\hline
\end{tabular}

Table 3 compares the contents of water extracts and alcohol extracts in leaves and seeds of Moringa. Compared with seeds, the water extracts and alcohol extracts in the leaves were higher than the seeds. The level of its content can be used as one of the evaluation indicators of the quality of Moringa [14].

\subsection{Determination of Total Polysaccharide and Flavonoids of Maringa Leaves.}

The calibration curves of glucose and rutin are shown in Fig.1a and Fig.1b, and their regression equation are $y=0.0754 x+0.0257(r=0.99965, n=6)$ and $y=0.264 x-0.007(r=0.99995, n=6)$, respectively.

According to the calibration curve, the content of total polysaccharide and total flavonoids obtained as showed in Table 4. As can be seen from Table 4, the total polysaccharide content up to $11.14 \%$, it is the key active ingredient of Moringa leaves and its content is an important quality evaluation index [3-4]. While the total flavonoids content is relatively low, less than 4\%, but flavonoids is an important active ingredient of Moringa leaves, with the function of preventing hypertension, hyperglycemia and hyperlipidemia, anti-oxidation and anti-tumor [1,9,11], and the level of its content plays an essential role in the quality evaluation of Moringa. 

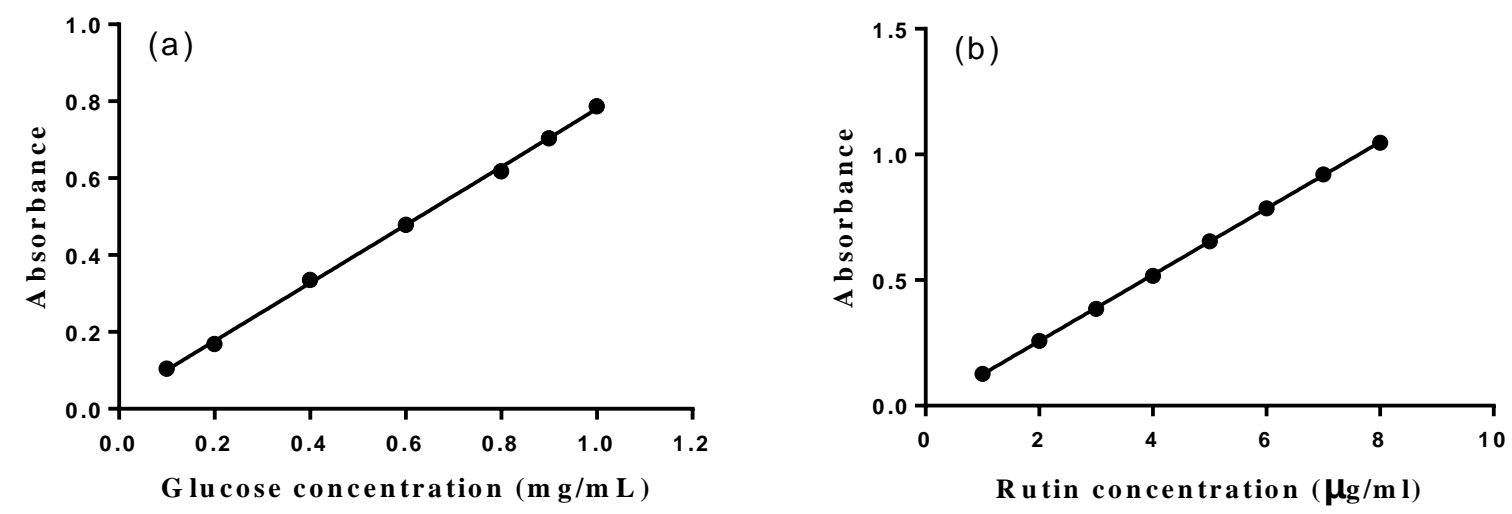

Figure 1. The calibration curve of glucose (a) and rutin (b)

Table 4. Content of total polysaccharide and total flavonoids of Moringa leaves $(n=6)$

\begin{tabular}{ccccccccc}
\hline \multirow{2}{*}{ Material } & \multicolumn{3}{c}{ Total polysaccharide } & & \multicolumn{3}{c}{ Total flavonoids } \\
\cline { 2 - 4 } \cline { 6 - 8 } \cline { 6 - 8 } & Mean $(\%)$ & SD & RSD (\%) & & Mean (\%) & SD & RSD (\%) \\
\hline Maringa leaves & 11.14 & 0.19 & 1.71 & & 3.70 & 0.05 & 1.24 \\
\hline
\end{tabular}

\subsection{Determination of Oil of Maringa Seeds.}

Table 5 shows the results of determination of the oil content of Moringa seeds. It can be seen from the table that Moringa seeds has a high oil content, close to $30 \%$, and its chemical composition is mostly monounsaturated fatty acids, accounting for about 76\% [12], which makes Moringa seeds oil has stable properties and is not easily corrupted, it is an excellent raw material for cosmetics, spices, and preservatives [16]. In addition, eating Moringa seeds oil can protect the liver, prevent liver cirrhosis hangover, anti-oxidation, anti-gastric ulcer, hypoglycemic and so on [16], so its level can also be used as an indicator of quality evaluation of Moringa seeds.

Table 5. Content of oil of Moringa seeds ( $n=6)$

\begin{tabular}{cccc}
\hline Material & Mean $(\%)$ & SD & RSD (\%) \\
\hline Moringa seeds & 22.70 & 0.50 & 2.22 \\
\hline
\end{tabular}

\section{Conclusion}

The contents of moisture, ash, water-extracts, alcohol-extracts and oil of leaves and seeds of Panzhihua Moringa have been tested. The results showed that the moisture content of leaves and seeds were $4.19 \%$ and $3.47 \%$, the total ash content were $11.04 \%$ and $3.57 \%$, the acid insoluble ash content were $2.18 \%$ and $0.3 \%$, the water extracts content were $36.28 \%$ and $19.19 \%$; The alcohol extracts content were $24.85 \%$ and $13.70 \%$. Polysaccharides and flavonoids are the main active ingredients of Moringa leaves, their contents were $11.14 \%$ and 3.70 respectively. And the oil content of seeds was as high as $30 \%$. This study provides an experimental basis for the evaluation of the comprehensive quality characteristics of Panzhihua Moringa in China and the development of related products. Future work will also be necessary to identify the specific structure of various compounds in total flavonoids and oils.

\section{Acknowledgements}

Special thanks goes to Xinguo Zhang for his technical support and help with this article.

\section{References}

[1]. Liu C-F and Li G-H. Research Status and Development Prospect of Maringa [J]. Journal of Yunnan Tropical Crops Science \& Technology, 2002, 03: 20. 
[2]. Ramachandran C, Peter K V, Gopalakrishnan P K. Drumstick (Maringa oleifera): a multipurpose Indian vegetable [J]. Economic botany, 1980, 34(3): 276-283.

[3]. Liu C-F and Li G-H. The nutritional value of Moringa oleifera [J]. Tropical Agricultural Science \& Technology, 2004, 01: 4.

[4]. Liang $\mathrm{P}$ and Zhen R-Y. Study on Extraction and Antioxidant Activity of Water-soluble Polysaccharides from Maringa Oleifera [J]. Food Res Dev., 2013, 14: 25-29.

[5]. Ran A-X, He Y-F, Jin G-M et al. Study on extraction processing of polysaccharide in mooring oleifera [J]. Food Science and Technology, 2008, 05: 191-194.

[6]. National Pharmacopoeia Committee. Pharmacopoeia of People's Republic of China [M]. Part 1. Beijing: Chemical Industry Press, 2015: Appendix 171, 174, 176.

[7]. Pang L. Study on Optimization of Ultrasound-assisted Extraction of Polysaccharide from Maringa [J]. Jiangsu Agricultural Sciences, 2008, 04: 238-239.

[8]. Chen R-Q, Pang S-S, Wang Y-Z et al. Determination of Polysaccharide in Leaves of Maringa oleifera [J]. Lishizhen Medicine and Material Medical Research, 2007, 07: 1700-1701.

[9]. Chen R-Q, Zhu B-F, Wang Y-Z et al. Extraction and Hypoglycaemic effect of the Total Flavonoid From leaves of Maringa oleic era [J]. Journal of Food Science and Biotechnology, 2007, 04: 42-45.

[10]. Sun M-Y and Wang Y-M. Study on Extraction of Flavonoids from Maringa oleifera Leaves [J]. Journal of Shao Guan University, 2007, 12:88-92.

[11]. Chen R-Q, Pang S-S, Wang Y-Z. Study on extraction technology of total flavones from levees of Maringa oleic era by ethanol [J]. Food Research and Development, 2007, 04: 29-31.

[12]. Zoo Y, Wang S-F, Boa X-P et al. Contrast and Study on the Volatile Oil of Maringa Seed at Two Extraction Methods[J]. Journal of Food Industry, 2011, 03: 61-63.

[13]. Yu J-X. Study on extraction method and hypolipidemic effect of Maringa oleifera seed oil [D]. Kunming: Kunming Medical University, 2009: 14-33.

[14]. Siddhuraju P, Becker K. Antioxidant properties of various solvent extracts of total phenolic constituents from three different agro climatic origins of drumstick tree (Meringa oleifera Lam.) leaves [J]. Journal of agricultural and food chemistry, 2003, 51(8): 2144-2155.

[15]. Manzo or M, Anwar F, Iqbal T, et al. Physic-chemical characterization of Maringa concanensis seeds and seed oil[J]. Journal of the American Oil Chemists' Society, 2007, 84(5): 413-419.

[16]. Hamza a. Ameliorative effects of Meringa oleifera Lam seed extract on liver fibrosis in rats [J]. Food and Chemical Toxicology, 2010, 48(1): 345-355. 